ISSN 1515-7326, nº 11, Año 2012, pp. 27 a 57

\title{
El escepticismo ante las reglas replanteado
}

\author{
Riccardo Guastini*
}

(Traducción de Federico José Arena)

\section{Teoría}

\subsection{Ambigüedad de "interpretación"}

En el lenguaje jurídico el vocablo "interpretación" adolece de una múltiple ambigüedad.

(1) Primera ambigüedad: Con el vocablo "interpretación" se hace referencia algunas veces a la atribución de significado a un texto normativo ("El texto $\mathrm{T}$ significa S", o, lo que es lo mismo, "La disposición jurídica D expresa la norma N"), y otras veces a la inclusión de un caso particular en una clase de casos regulados por una norma ("El hecho F es un caso de robo") y que constituye el último tramo del proceso de decisión -o de justificación de la decisión- del caso bajo consideración ${ }^{1}$.

* Dipartimento Giovanni Tarello, Università di Genova, guastini@unige.it

${ }^{1}$ Véase R. Guastini, L'interpretazione dei documenti normativi, Giuffré, Milano, 2004, p. 82 y ss. 
Es bastante posible que en el razonamiento jurídico (y especialmente en el judicial), entendido como un proceso psicológico, el adscribir significado a un texto normativo y el subsumir un caso bajo una clase de casos no puedan ser claramente distinguidos. Quizás los jueces primero clasifican el caso bajo consideración y sólo después proceden a inspeccionar los textos normativos para atribuirles un significado que les permita encontrar una regla que encaje con ello ${ }^{2}$. Sin embargo, desde un punto de vista lógico, atribuir significado a un texto y clasificar casos son dos cuestiones, si bien relacionadas, claramente diferentes. Asimismo, la atribución de significado es lógicamente antecedente a la clasificación de casos. Una cosa es preguntarse sobre la norma (o normas) expresada(s) y/o implicada(s) ${ }^{3}$ por una disposición jurídica y otra cosa es considerar si una norma así identificada se aplica o no a un caso particular. Los teóricos del derecho suelen limitarse a la primera operación, mientras que los jueces, en cuanto órganos de aplicación del derecho, deben llevar a cabo ambas.

En consecuencia es necesario distinguir entre ${ }^{4}$ :

(i) la interpretación dirigida a textos (o "in abstracto"), que consiste en identificar el contenido de significado -esto es, el contenido

${ }^{2}$ Esta es una tesis realista clásica, tal como lo señala B. Leiter en "Legal Realism and Legal Positivism Reconsidered", en B. Leiter, Naturalizing Jurisprudence. Essays on American Legal Realism and Naturalism in Legal Philosophy, Oxford U.P., Oxford, 2007, p. 61: «the Realists all embraced the following descriptive thesis: in deciding cases, judges react primarily to the underlying facts of the case, rather than to the applicable legal rules and reasons (the latter figuring primarily as ways of providing post-hoc rationales for decisions reached on other grounds)». Véase también A. Ross, On Law and Justice, Stevens \& Sons, London, 1958, p. 152.

${ }^{3}$ No existe una distinción precisa entre aquello que una formulación normativa significa y aquello que es implicado por ese significado. C.E. Alchourrón, E. Bulygin, Normative Systems, Springer, Wien-New York, 1971, pp. 67 f.: «The description of the law [...] involves the operation which jurists refer to under the vague term "interpretation" and which fundamentally consists in the determination of the consequences that can be derived from [...] norms».

${ }^{4}$ Una distinción similar es presentada por A. Aarnio, Philosophical Perspectives in Jurisprudence, Acta Philosophica Fennica, vol. 36, Helsinki, 1983, p. 165. Véase 
normativo (la norma o las normas)- expresado por, y/o lógicamente implícito en, un texto normativo, sin hacer referencia a algún caso concreto; y

(ii) la interpretación dirigida a hechos (o "in concreto") que consiste en subsumir un caso concreto en el campo de aplicación de una norma previamente identificada "in abstracto".

No es necesario, supongo, suministrar ejemplos de los problemas que presenta la interpretación dirigida a hechos (¿La regla "Prohibido vehículos en el parque" se aplica a las bicicletas o no?, ¿La regla constitucional referida a la "libertad de religión" se aplica a la denominada filosofía new-age o no?, etc.). Por el contrario, creo necesario señalar algunos ejemplos de los problemas que presenta la interpretación dirigida a textos.

(a) El artículo 13 de la Constitución francesa establece: "El Presidente de la República firma los decretos y resoluciones emanados del Consejo de Ministros". ¿Hemos de asumir que el Presidente posee el poder de firmar o que tiene el deber de hacerlo? ${ }^{5}$

(b) El artículo 11 de la Constitución italiana autoriza a la República a aceptar "aquellas limitaciones a la soberanía que sean necesarias para el establecimiento de un orden mundial que asegure la paz y la justicia entre Naciones.” ¿Cuál es el significado de "soberanía” en este contexto? ¿Se refiere, a grandes rasgos, a la independencia del Estado en las relaciones internacionales o al poder político supremo mencionado en el artículo 1, según el cual "la soberanía pertenece al pueblo"? ${ }^{6}$ En otras palabras, ¿hemos de asumir que el artículo 11

también W. Twining, D. Miers, How to Do Things with Rules. A Primer of Interpretation, 2da. ed., Weidenfeld and Nicholson, London, 1982, cap. 4, donde se distingue entre interpretación y aplicación.

5 Véase M. Troper, "La signature des ordonnances: fonctions d'une controverse", en M. Troper, Pour une théorie juridique de l'Ėtat, P.U.F., París, 1994, cap. XVIII.

${ }^{6}$ R. Guastini, "La primauté du droit communautaire: une révision tacite de la Constitution italienne", en Les Cahiers du Conseil Constitutionnel, 9, 2000, p. 119 y ss. 
autoriza limitaciones (únicamente) a la soberanía internacional del Estado o (también) al poder político supremo del pueblo?

(c) El artículo 2 de la Constitución italiana proclama solemnemente la "inviolabilidad de los derechos del hombre". ¿Qué significa "inviolabilidad"? ¿Se trata meramente de un fragmento de "retórica constitucional"7 que no expresa regla alguna? ¿Estamos frente a la simple reiteración del carácter "rígido" de la constitución (los derechos constitucionales no pueden ser violados por la ley ordinaria)? ¿O quiere decir que tales derechos no pueden ni siquiera ser objeto de una revisión constitucional (ni siquiera mediante el procedimiento de reforma constitucional)? ${ }^{8}$

La interpretación dirigida a textos se parece a la traducción ${ }^{9}$, dado que consiste en la identificación del sentido (Sinn, en palabras de Frege) de un texto y en la reformulación (rewording) del texto interpretado ${ }^{10}$. La interpretación dirigida a hechos, en cambio, no es otra cosa que una decisión acerca de la extensión o referencia (Bedeutung) de un concepto con relación a un caso concreto.

El objeto de la interpretación orientada a textos consiste en enunciados normativos (completos), mientras que la interpretación orientada a hechos recae sobre predicados en sentido lógico, es decir,

7 Tomo la expresión de L. Prieto Sanchís, Constitucionalismo y positivismo, Fontamara, México, 1997, p. 33.

${ }^{8}$ Esta es la opinión de la Corte Constitucional italiana (Corte Costituzionale, decisión 1086/1988). El tema es extensamente analizado en R. Guastini, "Esercizi d'interpretazione dell'art. 2 cost.", en Ragion pratica, 29, December 2007, p. 325 y ss.

${ }^{9}$ U. Eco, Dire quasi la stessa cosa. Esperienze di traduzione, Bompiani, Milano, 2003, cap. X; A. Marmor, Positive Law and Objective Values, Clarendon, Oxford, 2001, p. 76.

${ }^{10}$ Por ejemplo, «Las resoluciones y los decretos emanados del Consejo de Ministros han de ser firmados por el Presidente de la República» significa que el Presidente tiene la obligación de firmar; «La República reconoce y garantiza los derechos inviolables del hombre» significa que las disposiciones constitucionales que atribuyen los derechos mencionados no son susceptibles de reforma constitucional, etc. 
El escepticismo ante las reglas replanteado

términos que denotan clases. Mediante la interpretación orientada a textos se identifican normas jurídicas (y clases de casos), mientras que mediante la interpretación orientada a hechos se contribuye a identificar los casos concretos regulados por cada norma.

(2) Segunda ambigüedad: Con el vocablo "interpretación" se hace referencia algunas veces a un acto de puro conocimiento, y otras veces a un acto de decisión, e incluso otras veces a un acto de creación normativa. En consecuencia deberíamos distinguir también entre ${ }^{11}$ :

(i) La interpretación cognitiva, que consiste en identificar, sin elegir entre ellos, los posibles y diferentes significados de un texto normativo (los significados admisibles según las reglas lingüísticas -sintácticas, semánticas y pragmáticas- compartidas, las distintas técnicas interpretativas en uso, y las tesis dogmáticas difundidas en doctrina) ${ }^{12}$;

(ii) La interpretación decisoria, que consiste en elegir un determinado significado entre los significados identificados (o identificables) por medio de la interpretación cognitiva, descartando los restantes ${ }^{13}$;

(iii) La interpretación creativa, que consiste en atribuir a un texto un significado "nuevo" no comprendido entre los significados

${ }^{11} \mathrm{La}$ distinción se inspira en H. Kelsen, Introduction to the Problems of Legal Theory (1934), trad. de B. Litschevski Paulson y S.L. Paulson, Clarendon Press, Oxford, 1992, p. 80 y s.; H. Kelsen, Pure Theory of Law (1960), trad. de M. Knight, University of California Press, Berkeley-Los Angeles-London, 1967, p. 353 y ss.

${ }^{12}$ H. Kelsen, Pure Theory of Law, cit., p. 355: «Jurisprudential [i.e., cognitive] interpretation can do no more than exhibit all possible meanings of a legal norm. Jurisprudence as cognition of law cannot decide between the possibilities exhibited by it, but must leave the decision to the legal organ who $[\ldots]$ is authorized to apply the law».

${ }^{13} \mathrm{H}$. Kelsen, Pure Theory of Law, cit., p. 354: «In the application of law by a legal organ, the cognitive interpretation of the law to be applied is combined with an act of will by which the law-applying organ chooses between the possibilities shown by cognitive interpretation». 
identificados (o identificables) por medio de la interpretación cognitiva $^{14}$.

Supongamos que una cierta disposición jurídica $\mathrm{D}$ es ambigua en cuanto puede ser interpretada como expresando tanto la norma N1 como la norma N2. La interpretación puramente cognitiva asumiría la forma de un enunciado del tipo "D puede significar o bien N1 o bien $\mathrm{N} 2$ "15, la interpretación decisoria, a su vez, se expresaría mediante una enunciado o bien del tipo "D significa N1", o bien del tipo "D significa N2"; la interpretación creativa, a su vez, consistirá en decir, por ejemplo, "D significa N3" (adviértase que por hipótesis N3 no está entre los significados posibles de $\mathrm{D}$, identificados mediante interpretación cognitiva).

La interpretación cognitiva tal como lo sugiere el mismo nombre, es una operación puramente cognoscitiva privada de cualquier efecto práctico ${ }^{16}$. La interpretación decisoria y la interpretación creativa, en cambio, son operaciones "políticas" 17 , que pueden ser llevadas a cabo tanto por un órgano de aplicación como por un jurista. La única

${ }^{14} \mathrm{H}$. Kelsen, Pure Theory of Law, cit., p. 354: «By way of authentic interpretation (that is, interpretation of a norm by the law-applying organ), not only one of the possibilities may be realized that have been shown by the cognitive interpretation [...]; but also a norm may be created which lies entirely outside the frame of the norm to be applied». Véase también H. Kelsen, The Law of the United Nations, Stevens \& Sons, London, 1950, p. xv.

${ }^{15} \mathrm{H}$. Kelsen, Introduction to the Problems of Legal Theory, cit., p. 80: «If 'interpretation' is understood as discovering the meaning of the norm to be applied, its result can only be the discovery of the frame that the norm to be interpreted represents and, within this frame, the cognition of various possibilities for application. Interpreting a statute, then, leads not necessarily to a single decision as the only correct decision, but possibly to a number of decisions, all of them of equal standing».

${ }^{16} \mathrm{H}$. Kelsen, Pure Theory of Law, cit., p. 355: «The interpretation of law by the science of law (jurisprudence) must be sharply distinguished [...] from the interpretation by legal organs. Jurisprudential interpretation is purely cognitive ascertainment of the meaning of legal norms».

${ }^{17}$ H. Kelsen, Pure Theory of Law, cit., p. 355 y s.: «An attorney who, in the interest of his client, propounds to the judge only one of several possible 
diferencia importante reside en el hecho de que la interpretación realizada por un órgano de aplicación es, en sentido kelseniano, "auténtica", es decir, es vinculante en cuanto está provista de consecuencias jurídicas (de las que, por el contrario, la interpretación ofrecida por los juristas, carece $)^{18}$.

Me interesa destacar, sin embargo, que la interpretación creativa ${ }^{19}$, tal como ha sido aquí definida, es un fenómeno algo raro. En la mayoría de los casos, la interpretación creativa asume una forma ligeramente distinta, consistente en extraer del texto normas no expresas (dichas "implícitas", en un sentido amplio no estrictamente lógico) mediante una amplia variedad de argumentos no deductivos (e.g., a contrariis, a simili, etc.). Dicha operación (la "construcción" de normas no expresas) no es, estrictamente hablando, un acto de "interpretación", se trata de un genuino acto de creación normativa -una verdadera forma de "legislación intersticial" llevada a cabo por los intérpretes- que podríamos denominar "construcción jurídica". Esta última anotación nos conduce a la tercera ambigüedad.

(3) Tercera ambigüedad: Con el vocablo "interpretación" se suele hacer referencia a las operaciones de los juristas y de los jueces en su

interpretations [...], or a writer who in his commentary extols a specific interpretation among many possible ones as the only "correct" one, does not render a function of legal science, but of legal politics». Véase también $\mathrm{H}$. Kelsen, Introduction to the Problems of Legal Theory, cit., p. 82.

${ }^{18}$ H. Kelsen, Pure Theory of Law, cit., p. 354: «The interpretation by the lawapplying organ is always authentic. It creates law [...]». Véase también M. Troper, "La liberté d'interprétation du juge constitutionnel", en M. Troper, $\mathrm{La}$ théorie du droit, le droit, l'État, P.U.F., París, 2001, p. 85 y ss.

${ }^{19}$ Permítaseme proporcionar un ejemplo de interpretación "creativa" extraído del derecho constitucional italiano. Una disposición constitucional se refiere a "las leyes en materia constitucional". La frase es evidentemente vaga pero no ambigua: en el uso común de los juristas significa lo mismo que "leyes ordinarias que regulan materias de importancia constitucional" (un ejemplo paradigmático son las leyes que regulan el sistema electoral del Parlamento). Sin embargo, la Corte Constitucional construye el significado de la frase como "leyes constitucionales", es decir, como leyes aprobadas mediante el 
conjunto. Sin embargo, es importante advertir que entre todas esas operaciones existen diferencias importantes. Es necesario distinguir entre:

(i) La interpretación propiamente dicha, es decir, la atribución de significado a textos normativos (en alguno de los sentidos y modos que he señalado más arriba).

(ii) La "construcción jurídica", esto es, las numerosas operaciones en las que por lo general consiste el trabajo de los juristas (aunque también de los jueces) y que sería difícil listar aquí de modo exhaustivo. Por ejemplo las conjeturas acerca de la denominada ratio legis, las hipótesis contrafácticas acerca de la intención del legislador, la creación de jerarquías axiológicas entre normas, la construcción de reglas no expresas, la concreción de principios abstractos, la ponderación entre principios (especialmente constitucionales) en conflicto, etc.

Entre todas estas operaciones, la construcción de normas no expresas posee especial importancia. Con "normas no expresas" me refiero a aquellas normas que no han sido formuladas por autoridad normativa alguna, normas que no pueden ser consideradas como uno de los contenidos de significado o como la consecuencia directa de una determinada disposición.

Toda norma no expresa es el resultado de un argumento en el que alguna de sus premisas es una norma expresa y la norma no expresa es la conclusión. Pero nótese que en la mayoría de los casos tales argumentos, primero, no son lógicamente válidos $\mathrm{y}$, segundo (y más importante), incluyen premisas que no son reglas expresas, sino esquemas conceptuales y teorías elaboradas arbitrariamente por la dogmática ${ }^{20}$.

Algunos ejemplos permitirán aclarar el punto:

(a) El artículo 139 de la Constitución italiana prohíbe cualquier revisión (incluso mediante proceso de reforma constitucional) de la "forma republicana" del Estado. La mayoría de los constitucionalistas, sin

procedimiento especial reservado para las modificaciones constitucionales (véase Corte Costituzionale, decisión 168/1963).

${ }^{20}$ R. Guastini, L'interpretazione dei documenti normativi, cit., p. 104 y ss. 
El escepticismo ante las reglas replanteado

embargo, asume que un estado republicano es, por definición, un estado democrático y concluye que tampoco está permitida la revisión de la forma democrática del Estado. Puede claramente advertirse que esta conclusión, cuyo único fundamento es un concepto dogmático de "república" cuanto menos controvertido, posee el notable efecto de impedir la revisión constitucional de casi todo el texto constitucional.

(b) La Corte de Justicia de la Comunidad Europea asume, por un lado, que el derecho de la Comunidad Europea y el derecho de los Estados miembros forman un sistema jurídico unificado y, por otro lado, que el derecho europeo es superior al derecho estatal. Sobre esta base concluye que la legislación estatal es inválida (o, en todo caso, no aplicable) cuando sea incompatible con el derecho comunitario ${ }^{21}$. Queda claro que ninguna de estas asunciones posee una contraparte textual en el tratado de la CEE.

(c) Según la teoría constitucionalista "clásica", surgida de la Ilustración, la función de toda constitución es limitar el poder político ${ }^{22}$. Este modo de ver implica que las reglas constitucionales están dirigidas (solo) a los órganos supremos del Estado y que de ningún modo están sujetas a aplicación por parte de los tribunales. Actualmente, sin embargo, la mayoría de los constitucionalistas piensan que la función de la constitución es (también o esencialmente) la de modelar las relaciones sociales entre los ciudadanos. A partir de esto concluyen que las reglas constitucionales deben ser aplicadas directamente por todos los jueces y en toda controversia (lo que en la dogmática alemana se denomina "Drittwirkung") ${ }^{23}$.

${ }^{21}$ Corte Europea de Justicia, 15 de julio de 1964, Caso 6/64, Costa. Véase también Corte Europea de Justicia, 9 de marzo de 1978, Caso 106/77, Simmenthal.

${ }^{22}$ Véase el artículo 16 de la Déclaration des droits de l'homme et du citoyen: «Toute société dans laquelle la garantie des droits n'est pas assurée, ni la séparation des pouvoirs déterminée, n'a point de constitution».

${ }^{23}$ Véase, e.g., G. Zagrebelsky, Il diritto mite. Legge, diritti, giustizia, Einaudi, Torino, 1992. 
La construcción de normas no expresas permite que los intérpretes actúen disimuladamente como legisladores. Y, de hecho, constituye la parte principal y más importante del trabajo de los juristas.

\subsection{Dos tipos de indeterminación jurídica}

La interpretación dirigida a textos y la interpretación dirigida a hechos, respectivamente, responden a dos tipos distintos de indeterminación que afectan al derecho ${ }^{24}$.

(1) En primer lugar -la indeterminación del sistema jurídico como tal- el derecho es indeterminado en el sentido que no está determinado cuáles son las normas expresadas por las fuentes legales y, en este sentido, que pertenecen al sistema jurídico ${ }^{25}$.

(2) En segundo lugar -la indeterminación de cada norma particular-el derecho es indeterminado en el sentido que no están determinados cuáles casos caen bajo el ámbito de aplicación de cada norma.

\subsection{Las fuentes de la indeterminación}

(1) La indeterminación del sistema jurídico como tal depende de la equivocidad (o ambigüedad en un sentido amplio no técnico) de los textos normativos $^{26}$. Por ejemplo:

${ }^{24}$ R. Guastini, L'interpretazione dei documenti normativi, cit., p. 79.

${ }^{25} \mathrm{El}$ tipo de indeterminación que tengo en mente (indeterminación interpretativa) no debe confundirse con la indeterminación que resulta de la vaguedad del criterio de validez o pertenencia (la así llamada regla de reconocimiento en términos hartianos). Una cosa es preguntarse si un texto dado expresa una cierta norma $\mathrm{N}$ o no, y otra cosa es preguntarse si $\mathrm{N}$ (asumiendo que de hecho es expresada por el texto en cuestión) es válida o no (por ejemplo, si es compatible o no con la Constitución).

${ }^{26} \mathrm{La}$ ambigüedad es la propiedad de aquellas expresiones que son capaces de expresar más de un significado (que son susceptibles de múltiples interpretaciones). Por lo tanto, un texto ambiguo, en primer lugar, no es un texto sin significado y, en segundo lugar, no es un texto que admita cualquier interpretación. Es importante recordar estas precisiones cuando se discute acerca del escepticismo radical ante las reglas. 
El escepticismo ante las reglas replanteado

(a) A veces un texto normativo $\mathrm{T}$ es, en sentido estricto, ambiguo por razones sintácticas o semánticas, es decir, dudamos si expresa la norma N1 o la norma N2 ${ }^{27}$.

(b) A veces todos están de acuerdo con que el texto normativo $\mathrm{T}$ expresa la norma N1, pero se duda si también expresa la norma N2 o no.

(c) A veces todos están de acuerdo con que el texto normativo $\mathrm{T}$ expresa la norma N1, pero se duda si esa norma implica o no la norma N2.

(d) A veces todos acuerdan con que el texto normativo $\mathrm{T}$ expresa la norma N1, pero se duda si la norma es derrotable (i.e., sujeta a excepciones implícitas) o no ${ }^{28}$.

Permítaseme ofrecer algunos ejemplos extraídos del derecho constitucional italiano.

Caso (a). Según la Constitución italiana, las leyes pueden ser derogadas mediante referéndum. Una disposición legal, sin embargo, establece que el referéndum derogatorio no es admisible "en el año anterior al fin del ejercicio legislativo de una de las dos Cámaras”. ¿Qué significa "año" en este contexto? ¿Significa 365 días (en francés: an)? ¿O significa el período que va desde el $1^{\circ}$ de enero al 31 de diciembre (en francés: anée)? Supongamos que las Cámaras concluyen el primero de mayo de 2010. ¿Debemos concluir que (N1) el referéndum no es admisible desde el primero de mayo de 2009 hasta el 31 de abril de 2010 o debemos concluir que (N2) el referéndum no es admisible durante todo el 2009?

Caso (b). Una disposición legislativa establece que no es admisible un nuevo referéndum derogatorio con relación a una ley respecto de la cual haya fracasado un referéndum anterior. Ahora bien, según la

${ }^{27}$ A. Ross, On Law and Justice, cit., p. 123 y ss.

${ }^{28}$ C.E. Alchourrón, "On Law and Logic", en Ratio Juris, 9, 1996. Véase también W. Twining, D. Miers, How to Do Things with Rules, cit., p. 216 y s. sobre "implied exceptions"; F. Schauer, Playing by the Rules. A Philosophical Examination of Rule-Based Decision-Making in Law and in Life, Clarendon, Oxford, 1991, p. 115 y s. 
Constitución un referéndum puede fracasar de dos maneras diferentes, a saber, o bien porque la mayoría votó "no", o bien porque la mayoría no votó. No hay dudas que un nuevo referéndum es inadmisible en aquellos casos en que la mayoría votó "no" (N1) pero ¿resulta igualmente inadmisible un nuevo referéndum en aquellos casos en los que la mayoría no votó (N2)?

Caso (c). La Constitución establece expresamente que el Parlamento puede proponer un voto de censura contra el Gobierno (N1). ¿Implica esto que el Parlamento puede también proponer un voto de censura contra cada uno de los ministros individualmente (N2)? ${ }^{29}$

Caso (d). La Constitución italiana otorga al Presidente de la República el poder de veto sobre las leyes. Sin dudas, esto es así con relación a la legislación ordinaria (N1). ¿Pero sucede o no lo mismo con relación a las "leyes constitucionales", es decir, las leyes aprobadas siguiendo un procedimiento especial y dirigidas a producir modificaciones constitucionales (excepción implícita)?

La interpretación dirigida a textos responde a preguntas de este tipo $\mathrm{y}$, al responderlas, reduce la indeterminación del sistema jurídico. El escepticismo ante las reglas, tal como lo concibo, equivale a sostener que cualquier tesis sobre la existencia de una única interpretación correcta -cualquier tesis que sostenga que los problemas interpretativos admiten una única respuesta "correcta" (i.e. verdadera)- es falsa. Y es falsa precisamente debido a la indeterminación del sistema jurídico en el sentido especificado, lo que trae como consecuencia que los enunciados interpretativos no tienen valor de verdad. La indeterminación interpretativa del sistema jurídico es el verdadero fundamento del escepticismo ante las reglas.

(2) La indeterminación de cada norma depende de la vaguedad o textura abierta ${ }^{30}$ de todo predicado del lenguaje natural ${ }^{31}$.

${ }^{29}$ La pregunta fue respondida de manera afirmativa por la Corte Constitucional italiana (Corte Costituzionale, decisión 6/1996).

${ }^{30}$ No es necesario aquí distinguir entre vaguedad y textura abierta.

${ }^{31}$ Las más exahustivas investigaciones sobre este tema son: C. Luzzati, La vaghezza delle norme. Un'analisi del linguaggio giuridico, Giuffré, Milano, 1990 y 
Respecto de cualquier texto normativo existen casos claros o fáciles que claramente caen bajo el núcleo de referencia de los términos usados en el texto, de tal forma que dicha norma es ciertamente aplicable a ellos, y existen también casos marginales o difíciles que caen en el área de penumbra que rodea el núcleo de referencia de los términos, de tal forma que la aplicabilidad de la norma a dichos casos es incierta o discutible ${ }^{32}$.

La interpretación dirigida a hechos responde este tipo de dudas y, de este modo, reduce la indeterminación de las normas.

La indeterminación de las normas provee también un fundamento al escepticismo ante las reglas. Se trata, sin embargo, de un fundamento menos importante dado que, desde mi punto de vista, la indeterminación de las normas se presenta solo una vez que ha tenido lugar la interpretación dirigida a textos, esto es, luego de que las normas han sido identificadas, y pone serios problemas solo en los casos difíciles. Los problemas de la interpretación dirigida a textos (y que dan lugar a la indeterminación interpretativa del sistema jurídico), en cambio, son mucho más invasivos.

\subsection{Disposiciones y normas}

La indeterminación del sistema jurídico impone distinguir cuidadosamente entre, por un lado, los enunciados normativos (formulaciones de normas o, como es común decir, las "disposiciones") contenidos en las fuentes del derecho y, por otro lado, su significado i.e. las normas expresas y/o implicadas ${ }^{33}$. Esto es así por tres razones principales:

T.A.O. Endicott, Vagueness in Law, Oxford U.P., Oxford, 2000. Ambas desarrollan un gran número de distinciones relevantes que, sin embargo, no es necesario traer a colación aquí.

${ }^{32}$ Véase H.P. Grice, Studies in the Way of Words, Harvard U.P., Cambridge (Mass.), 1989, p. 177.

${ }^{33}$ R. Guastini, "Rules, Validity, and Statutory Construction", en M. Jori, A. Pintore (eds.), Law and Language. The Italian Analytical School, Deborah Charles Publication, Liverpool, 1997. 
(i) Primero, muchos enunciados normativos son ambiguos (en sentido estricto), es decir, expresan alternativamente dos (o más) normas.

(ii) Segundo, muchos enunciados normativos (quizás todos ellos) poseen un contenido de significado complejo, en el sentido que expresan y/o implican no una única norma, sino una pluralidad de ellas.

(iii) Tercero, según el uso común de los juristas, cada uno y todos los sistemas jurídicos están repletos de normas "no expresas". Esto es, normas que no corresponden a ningún enunciado normativo en particular, puesto que no han sido formuladas por autoridad normativa alguna $^{34}$.

\subsection{Las fuentes principales de indeterminación jurídica}

Es importante advertir que la indeterminación del derecho (sobre todo, la indeterminación del sistema jurídico como tal) no depende solo de ciertos defectos "objetivos" del lenguaje constitucional o legislativo. Las fuentes principales de la indeterminación ${ }^{35}$, en cambio, son:

(i) la multiplicidad de métodos interpretativos ${ }^{36}$;

(ii) la dogmática jurídica ${ }^{37}$, y

(iii) el sentimiento de justicia de los intérpretes, i.e., sus preferencias éticas y políticas (las declaren o no mediante juicios de valor explícitos) ${ }^{38}$.

${ }^{34}$ Una cuarta, si bien menor, razón para distinguir entre enunciados normativos y normas es que dos enunciados normativos distintos pueden expresar una misma norma. Véase F. Schauer, Playing by the Rules, cit., p. 62 y s.

${ }^{35}$ Véase W. Twining, D. Miers, How to Do Things with Rules, cit., p. 218 y ss., sobre el "intérprete atribulado" y las "condiciones de duda" en la interpretación.

${ }^{36}$ "The set of interpretive operations that can be legitimately performed on [legal] sources (to generate rules and principles of law)»: J.L. Coleman, B. Leiter, "Determinacy, Objectivity, and Authority", en A. Marmor (ed.), Law and Interpretation. Essays in Legal Philosophy, Clarendon Press, Oxford, 1995, p. 213.

${ }^{37} \mathrm{G}$. Tarello, "La semantica del neustico. Osservazioni sulla 'parte descrittiva' degli enunciati precettivi", en G. Tarello, Diritto, enunciati, usi. Studi di teoria e metateoria del diritto, Il Mulino, Bologna, 1974, p. 329 y ss.

${ }^{38}$ Véase, por ejemplo, A. Ross, On Law and Justice, cit., p. 145 y s. 
La influencia que el sentimiento de justicia de los intérpretes ejerce en sus elecciones interpretativas es tan evidente que no creo necesario desarrollar este punto. En cambio, me parece necesario formular algunas consideraciones adicionales con relación a los métodos interpretativos y a la dogmática.

En primer lugar, en la mayoría de los casos un mismo enunciado normativo puede expresar significados diferentes según sea sometido a uno $\mathrm{u}$ otro método interpretativo ${ }^{39}$. Tomemos, por ejemplo, una disposición constitucional (de la Constitución italiana) que se refiera a las "leyes". Argumentando a contrario uno puede concluir que esa disposición es aplicable a todas las leyes y solo a las leyes. Argumentando por analogía, en cambio, se podría concluir que la disposición en cuestión es aplicable a las leyes y también a los reglamentos administrativos (dado que ambos son "fuente del derecho"). Argumentando mediante la técnica de la disociación ("distinguishing"), uno puede concluir que, dado que la clase de las leyes incluye diferentes subclases (constitucionales y ordinarias, por un lado; regionales y estatales, por el otro), la disposición se aplica -a la luz de su ratio- solo a uno de esos subconjuntos. De hecho, el conjunto de métodos interpretativos (generalmente aceptados en una comunidad jurídica dada) es suficiente para garantizar una amplia cantidad de resultados diferentes y rivales entre si $^{40}$.

${ }^{39} \mathrm{H}$. Kelsen, The Law of the United Nations, cit., p. xiii: «The different methods of interpretation may establish different meanings of one and the same provision. Sometimes, even one and the same method, especially the so-called grammatical interpretation, leads to contradictory results». Cf. K.N. Llewellyn, "Remarks on the Theory of Appellate Decisions and the Rules or Canons about how Statutes Are to be Construed» (1950), viz., el fragmento reimpreso en W.W. Fisher III, M.J. Horwitz, T.A. Reed (eds.), American Legal Realism, Oxford U.P., New York-Oxford, 1993, p. 228 y ss.; A. Ross, On Law and Justice, cit., p. 148 y ss.; G. Tarello, L’interpretazione della legge, Giuffré, Milano, 1980, p. 341 y s.; E. Diciotti, Interpretazione della legge e discorso razionale, Giappichelli, Torino, 1999, esp. cap. V; P. Chiassoni, Tecnica dell'interpretazione giuridica, Il Mulino, Bologna, 2007, cap. II.

${ }^{40}$ Cf. J.L. Coleman, B. Leiter, "Determinacy, Objectivity, and Authority", cit., p. 214. 
Segundo, todo intérprete se acerca a los textos legales provisto de una serie de presupuestos teóricos y conceptuales que, de manera inevitable, condicionan sus interpretaciones, ya sea orientándolas en una determinada dirección ya sea excluyendo otras interpretaciones que, de otro modo, podrían haber sido elegidas ${ }^{41}$. Tales presupuestos no son otra cosa que las teorías elaboradas por los juristas en un momento lógicamente antecedente a la interpretación de un texto determinado. Pienso aquí en teorías tales como la de la constitución escrita formulada por la Corte Suprema de Estados Unidos en el caso Marbury (toda constitución escrita implica el principio según el cual cualquier ley incompatible con la constitución es nula y vacía), la teoría de las relaciones entre el derecho de la Comunidad Europea y los sistemas jurídicos de cada estado miembro presupuesta por la Corte Europea de Justicia en numerosas decisiones (el derecho europeo y los sistemas jurídicos estatales son partes de un sistema jurídico unificado), la teoría del gobierno parlamentario presupuesta por la mayoría de los juristas continentales (teoría según la cual el Jefe de Estado es un poder neutral sin competencia alguna con relación a cuestiones estrictamente políticas), las distintas teorías de los contratos, de la propiedad, de la responsabilidad civil, etc.

\section{Metateoría}

\subsection{Tres teorías de la interpretación jurídica}

Se suele distinguir, en el pensamiento jurídico moderno, tres teorías rivales de la interpretación jurídica. Siguiendo a otros filósofos del derecho las llamaré respectivamente: la teoría del "noble sueño", la teoría de "la pesadilla" 42 y la teoría de "la vigilia" 43 . Las diferencias entre ellas son, a grandes rasgos, las siguientes.

${ }^{41}$ W. Twining, D. Miers, How to Do Things with Rules, cit., p. 338 y s.

${ }^{42}$ H.L.A. Hart, "American Jurisprudence through English Eyes: The Nightmare and the Noble Dream" (1977), en H.L.A. Hart, Essays in Jurisprudence and Philosophy, Clarendon, Oxford, 1983, p. 123 y ss.

43 J.J. Moreso, Legal Indeterminacy and Constitutional Interpretation, Kluwer, Dordrecht-Boston-London, 1998, p. 156 y ss.; M. Barberis, "Lo scetticismo 
(i) La teoría del noble sueño -o cognitivismo interpretativo, cuyos orígenes se remontan hasta las ideas jurídicas de la Ilustración (Montesquieu, Cesare Beccaria, etc.)- asume que cada texto normativo se encuentra provisto de un significado "objetivo", único y determinado, que es susceptible de verdad. De este modo, la interpretación jurídica se concibe como una actividad cognitiva que equivale a descubrir el único significado preexistente. En particular, por lo que se refiere a las decisiones judiciales, no existe espacio alguno para la genuina discreción interpretativa, en el sentido que las decisiones judiciales que se adhieren al significado objetivo del texto son correctas mientras que cualquier otra decisión no lo es.

(ii) La teoría de la pesadilla -una forma radical de escepticismo que puede ser atribuida a algunas versiones del realismo jurídico- asume que los textos normativos no tienen ningún significado antes de la interpretación ${ }^{44}$. El significado de los textos normativos es el resultado de la interpretación, no su objeto ${ }^{45}$. De este modo, la interpretación jurídica no es, bajo ningún punto de vista, una empresa cognitiva. Interpretar es un "acto de voluntad" y no de conocimiento $^{46}$, es decir, se trata de una cuestión de valores, de elecciones y de decisiones. Se sigue que, por lo que se refiere a las decisiones judiciales, la discreción interpretativa es inevitable y que

immaginario. Nove obiezioni agli scettici à la génoise", en P. Comanducci, R. Guastini (eds.), Analisi e diritto 2000. Ricerche di giurisprudenza analitica, Torino, 2001, p. 2 y ss.

${ }^{44}$ J.C. Gray, The Nature and Sources of the Law, 2da. ed. a partir de las notas del autor, R. Gray (ed.), Macmillan, New York, 1948, esp. pp. 124 y 170; M. Troper, "Une théorie réaliste de l'interprétation", en M. Troper, La théorie du droit, le droit, l'État, cit., p. 69 y ss.

45 G. Tarello, "Introduzione al linguaggio precettivo" e "Il "problema dell'interpretazione': una formulazione ambigua", ambos en G. Tarello, Diritto, enunciati, usi, cit., esp. 265 y ss., 389 y ss. Véase también R. Guastini, Dalle fonti alle norme, 2da ed., Giappichelli, Torino, 1992, esp. cap. VIII; T. Mazzarese, "Norm Proposition': Epistemic and Semantic Queries", en Rechtstheorie, vol. XXII, 1991, p. 39 y ss.; R. Guastini, "Fragments of a Theory of Legal Sources", en Ratio Juris, vol. IX, 1996.

${ }^{46} \mathrm{H}$. Kelsen, Introduction to the Problems of Legal Theory, cit., p. 82 y s. 
distinguir entre decisiones correctas e incorrectas simplemente no tiene ningún sentido. Toda interpretación avanzada por los jueces es "auténtica" (en sentido kelseniano), es decir, creadora de derecho.

(iii) Finalmente la teoría de la vigilia -que puede ser reconducida a Hart $^{47}$ - tiene una actitud mixta hacia la interpretación jurídica. Distingue entre casos fáciles y difíciles ${ }^{48}$. Los textos normativos, sostiene la teoría, están formulados en lenguaje natural usando "términos clasificatorios generales" (predicados en sentido lógico) y tales términos son fatalmente vagos o de textura abierta ${ }^{49}$. En consecuencia, dada una norma cualquiera, existen casos que seguramente caen bajo su ámbito de aplicación y casos que seguramente no lo hacen. Pero, junto a esos casos "fáciles", encontramos también casos "difíciles", casos en los que la aplicación de la norma es dudosa y discutible ${ }^{50}$. En los casos fáciles los jueces no poseen discreción interpretativa alguna, pero en los casos difíciles, en cambio, la interpretación elegida será el resultado de una decisión discrecional acerca de la extensión o referencia de los conceptos expresados por los términos generales. En los casos fáciles sí es posible distinguir entre interpretaciones jurídicamente correctas e interpretaciones jurídicamente incorrectas, pero ello no es posible en los casos difíciles.

Es necesario advertir que ninguna de las tres teorías mencionadas muestra algún tipo de interés por la interpretación de los juristas, todas

${ }^{47}$ H.L.A. Hart, "Positivism and the Separation of Law and Morals" (1958), en H.L.A. Hart, Essays in Jurisprudence and Philosophy, cit., esp. p. 64 y ss.; H.L.A. Hart, The Concept of Law, Clarendon, Oxford, 1961, cap. VII.

${ }^{48}$ G.R. Carrió, Notas sobre derecho y lenguaje, 4ta. ed., Abeledo-Perrot, Buenos Aires, 1994, p. 49 y ss.; E. Bulygin, "Cognition and Interpretation of Law", en L. Gianformaggio, S.L. Paulson (eds.), Cognition and Interpretation of Law, Giappichelli, Torino, 1995, p. 11 y ss.; J.J. Moreso, Legal Indeterminacy and Constitutional Interpretation, cit., p. 156 y ss. Véase también el análisis de las denominadas "lagunas de reconocimiento" en C.E. Alchourrón, E. Bulygin, Normative Systems, cit., p. 33 y s., 146.

${ }^{49}$ F. Waismann, "Verifiability', en A. Flew (ed.), Logic and Language, Blackwell, Oxford, 1952.

${ }^{50}$ H.L.A. Hart, The Concept of Law, cit., cap. VII. 
se concentran únicamente en la interpretación judicial ${ }^{51}$. Desde mi punto de vista esto constituye un serio defecto por dos razones principales. Primero, la interpretación de los juristas y la de los jueces no son necesariamente idénticas desde el punto de vista del análisis lógico (por ejemplo, en ningún caso la interpretación judicial puede ser meramente cognitiva y/o meramente dirigida a textos). Segundo, los jueces, antes de asumir su cargo, han frecuentado la facultad de derecho y esto implica que el pensamiento de los juristas -las teorías dogmáticas y los métodos interpretativos- condiciona las ideas que los jueces poseen acerca del derecho en general, de los conceptos jurídicos y de la interpretación judicial (de hecho, condiciona la propia forma mentis de los jueces).

\subsection{Una reconstrucción analítica}

Desde una perspectiva analítica estos diferentes puntos de vista pueden ser reconstruidos no como teorías psicológicas (¿la interpretación es un acto de voluntad o un acto de conocimiento ${ }^{52}$ ), sino como teorías semánticas, es decir, como teorías acerca del valor de verdad de los enunciados interpretativos ${ }^{53}$.

Por "enunciado interpretativo" entiendo, a grandes rasgos, todo enunciado que posee la forma lógica "T significa S" (donde T se refiere a un fragmento de texto normativo cualquiera -por ejemplo, una cláusula constitucional, una disposición legislativa, etc.- y S se refiere al significado adscripto).

(i) De acuerdo a la teoría del noble sueño todo enunciado interpretativo ha de ser o bien verdadero o bien falso ${ }^{54}$. Como

${ }^{51}$ W. Twining, D. Miers, How to Do Things with Rules, cit., p. 176.

${ }^{52}$ Este es el modo en el que Kelsen plantea la cuestión (H. Kelsen, Introduction to the Problems of Legal Theory, cit., pp. $82 \mathrm{f}$.).

${ }^{53}$ R. Guastini, "Interpretive Statements", en E. Garzón Valdés, W. Krawietz, G. H. von Wright, R. Zimmerling (eds.), Normative Systems in Legal and Moral Theory. Festschrift for Carlos E. Alchourrón and Eugenio Bulygin, Duncker \& Humblot, Berlin, 1997, p. 279 y ss.

${ }^{54}$ Esta tesis es expresamente defendida por R. Hernández Marín, Interpretación, subsunción, y aplicación del derecho, Marcial Pons, Madrid-Barcelona, 1999. 
señalé, este punto de vista presupone que los textos normativos se encuentran provistos de un único significado objetivo -ya sea el significado literal o el significado intencional- de modo tal que, para cada texto normativo, existe un único enunciado interpretativo que es verdadero, mientras que todos los demás son fatalmente falsos.

(ii) Según la teoría de la pesadilla, por el contrario, los enunciados interpretativos no poseen en ningún caso valor de verdad. Antes de la interpretación los textos legales no poseen ningún significado. E1 significado es una creación "libre", sin restricciones, de los intérpretes (es decir, los jueces).

(iii) De acuerdo con la teoría de la vigilia, en fin, es necesario distinguir entre dos tipos de enunciados interpretativos. En los casos fáciles el significado de los textos normativos puede (y debe) ser simplemente declarado, en los casos difíciles ha de ser decidido de manera discrecional. Esto equivale a decir que los enunciados interpretativos poseen valor de verdad cuando son proferidos en casos fáciles, mientras que no poseen valor de verdad cuando son proferidos en casos difíciles. Esto es así dado que los textos normativos poseen, de hecho, un significado objetivo pero lamentablemente ese significado es indeterminado (en particular, de textura abierta) y por lo tanto deja espacio para la discreción en los casos marginales ${ }^{55}$.

A continuación expondré los argumentos sobre la base de los cuales considero que ninguna de estas tres teorías es correcta.

\subsection{Teoría del significado y teoría de la interpretación}

Mi primer argumento se dirige contra toda forma de cognitivismo en el ámbito de la interpretación jurídica -la teoría del noble sueño se ve directamente afectada, pero también se ve afectada la teoría de la vigilia (en otro lugar he argumentado que la teoría de la vigilia no es más que una forma sofisticada de cognitivismo $)^{56}$.

55 G.R. Carrió, Notas sobre derecho y lenguaje, cit., p. 56 y ss.

${ }^{56}$ R. Guastini, "A Sceptical View on Legal Interpretation”, en P. Comanducci, 
Supongamos, en beneficio del argumento, que los textos normativos (las disposiciones constitucionales, las disposiciones legislativas, etc.) poseen un significado determinado y que, en consecuencia, los enunciados interpretativos pueden ser considerados verdaderos o falsos $^{57}$. E1 único modo plausible de defender una tesis tal es proveyendo algún criterio que permita determinar el valor de verdad de los enunciados interpretativos. Por otra parte, los enunciados interpretativos son enunciados que atribuyen significado, por lo que parece obvio que establecer las condiciones de verdad de un enunciado que atribuye significado exige una determinada teoría del significado. ¿Pero cuál?

En la historia del pensamiento jurídico es posible, sin mucho esfuerzo, encontrar dos candidatos principales ${ }^{58}$ (si bien sería posible identificar un número mayor de teorías implícitas analizando con mayor detenimiento los métodos de interpretación que son de hecho usados por los juristas):

(a) por un lado, la teoría del significado literal: el significado depende simplemente de -y puede ser descubierto aplicando- las reglas sintácticas y semánticas del lenguaje compartido por una comunidad lingüística dada;

(b) por el otro lado, la teoría intencionalista del significado (o teoría del significado intencional): el significado depende -y puede ser descubierto averiguando- la intención de la autoridad normativa,

R. Guastini (eds.), Analisi e diritto 2005. Ricerche di giurisprudenza analitica, Giappichelli, Torino, 2006, p. 139 y ss.; R. Guastini, "Lo scetticismo interpretativo rivisitato", en Materiali per una storia della cultura giuridica, 36, 2006, p. 227 y ss.

${ }^{57}$ Nótese que este modo de ver posee consecuencias paradójicas. Frente a las numerosas interpretaciones de un mismo texto uno debería concluir que solo una de ellas es verdadera y las restantes fatalmente falsas. ¿No resultaría de este modo sorprendente la enorme cantidad de interpretaciones falsas (aunque emitidas por juristas expertos) que se llevan a cabo en la práctica jurídica diaria?

${ }^{58}$ Véase, e.g., B. Bix, Jurisprudence: Text and Context, Sweet \& Maxwell, London, 1999, cap. XIV. 
esto es, del sujeto (un individuo o un órgano colegiado) que produjo el texto bajo análisis ${ }^{59}$.

Ahora bien, si se mira atentamente, estas dos teorías pueden ser entendidas de dos modos diferentes. Ya sea como teorías genuinamente descriptivas de aquello que el significado efectivamente es (más precisamente, como teorías acerca de cómo el significado es atribuido), o como teorías (si bien no abiertamente) normativas acerca de cómo el significado debe ser atribuido (cómo han de ser interpretados los textos normativos). En lo que sigue exploro ambas posibilidades.

Asumamos que las teorías jurídicas más comunes acerca del significado son descriptivas, es decir, que se limitan a afirmar en qué consiste, objetivamente, el significado. En ese caso, ¿sería alguna de ellas verdadera?

Para responder a esta pregunta, sin embargo, es necesario responder a otra pregunta preliminar, a saber, ¿en qué consiste una teoría del significado? Asumo que una teoría descriptiva del significado consiste simplemente en reconstruir las prácticas interpretativas de una determinada comunidad de intérpretes, por ejemplo, de los hablantes del español (del italiano, del francés, etc.) en sus conversaciones diarias, cuando leen novelas o periódicos, escuchan la radio, etc. ${ }^{60}$

Ahora bien, cada una de las dos teorías del significado mencionadas probablemente describe (una parte significativa de) la práctica de atribución de significado en la conversación ordinaria. Pero es evidente que ninguna de ellas describe la práctica de los intérpretes jurídicos, se trate de jueces, abogados o juristas.

Es un hecho que los intérpretes -incluso uno y el mismo intérprete- se atienen alternativamente al uso ordinario, a la (supuesta) intención del legislador, a la denominada "ratio legis", al significado que (presumiblemente) se obtiene si se considera el sistema jurídico como un

${ }^{59}$ Acerca del posible papel de intenciones, motivos, razones y propósitos en la interpretación véase W. Twining, D. Miers, How to Do Things with Rules, cit., p. 192 y ss.

${ }^{60}$ P. Chiassoni, "Interpretive Games. Statutory Construction through Gricean Eyes", en P. Comanducci, R. Guastini (eds.), Analisi e diritto 1999. Ricerche di giurisprudenza analitica, Torino, 2000, p. 86 y ss. 
todo, etc. De hecho, los juristas han construido a lo largo del tiempo, una gran cantidad de instrumentos conceptuales y argumentativos que les permiten elegir, respecto de un mismo texto, entre un (a veces amplio) conjunto de significados posibles.

Por lo tanto, en cuanto referidas al lenguaje jurídico, las dos teorías del significado mencionadas -tal como, creo, cualquier otra teoría del significado que se les parezca- son de manera evidente ambas falsas y, me atrevería a decir, ingenuas.

Son falsas, claro está, si se las concibe como genuinas teorías descriptivas, ya que solo las teorías descriptivas pueden ser verdaderas o falsas. Existe, sin embargo otra posibilidad, a saber, ambas teorías pueden (y quizás deben) ser entendidas como teorías normativas de la interpretación. Es decir, como teorías con la pretensión de ex ante dirigir $\mathrm{y}$ ex post criticar la práctica de atribución de significado de los juristas $\mathrm{y}$, sobre todo, de los jueces. ¿Existe una diferencia relevante, en contextos jurídicos, entre afirmar que el significado depende de las reglas del lenguaje ordinario y recomendar que los intérpretes atribuyan significado a un texto según esas reglas? ¿Cuál es el sentido de afirmar, al menos en contextos jurídicos, que el significado depende de la intención de la autoridad normativa, sino el sugerir que los intérpretes deben descubrir esa intención y atenerse a ella?

Ahora bien, ¿qué hay de malo en las teorías normativas del significado? Nada, claro. Salvo que ellas no son de ayuda para describir qué es en realidad la interpretación.

Si lo que digo es correcto, se sigue una importante consecuencia. Una teoría descriptiva de la interpretación jurídica no necesita una teoría del significado previa. Ello se debe a dos razones.

Una teoría descriptiva de la interpretación no necesita una teoría normativa del significado ya que prescribir no forma parte de su cometido $^{61}$. Orientar y/o evaluar la práctica interpretativa de los juristas y de los jueces es seguramente una empresa valiosa, pero no es una empresa cognitiva. Evocando una tesis realista puede decirse que "la

${ }^{61}$ H.L.A. Hart, The Concept of Law, 2da. ed., Clarendon, Oxford, 1994, Postscript, p. 243 y s. 
tarea de la teoría del derecho [es] identificar y describir -no justificar- los patrones" del pensamiento jurídico ${ }^{62}$.

Sobre todo, una teoría descriptiva de la interpretación no necesita una teoría descriptiva del significado -o mejor, una teoría descriptiva de la interpretación no puede presuponer ninguna determinada teoría descriptiva del significado- puesto que una teoría descriptiva de la interpretación es ella misma una teoría descriptiva del significado en contextos jurídicos (y, en todo caso, la única teoría del significado admisible en contextos jurídicos).

¿En qué puede consistir una teoría puramente descriptiva del significado sino en la reconstrucción de la práctica efectiva de una determinada comunidad interpretativa? La teoría del significado referida a textos jurídicos no puede ser otra cosa más que el análisis y la reconstrucción racional de la práctica de atribución de significado llevada a cabo por los intérpretes jurídicos. Por lo tanto, no se diferencia de una teoría de la interpretación, es la misma cosa.

\subsection{Los límites (conceptuales) de la interpretación}

La teoría de la pesadilla, tal como la he presentado, es una forma radical de escepticismo. Según esta teoría anything goes en materia interpretativa, y ello es así por una razón de tipo Humpty-Dumpty ${ }^{63}$, a saber, que "whoever hath an absolute authority to interpret any written or spoken laws, it is he who is truly the Law-giver to all intents and purposes, and not the person who first wrote or spoke them" 64 .

Entendida como una descripción del contenido y del modo de funcionar de (la mayoría de) los sistemas jurídicos actuales esta tesis es bastante correcta. Es un hecho que los jueces de última instancia, como así también otras autoridades interpretativas cuyas decisiones no pueden

${ }^{62}$ B. Leiter, "Legal Realism and Legal Positivism Reconsidered", cit., p. 63 (refiriéndose a la opinión de Herman Oliphant).

${ }^{63}$ U. Scarpelli, "Il metodo giuridico", en U. Scarpelli, L'etica senza verità, Il Mulino, Bologna, 1982, p. 179 y ss.

${ }^{64}$ Benjamin Hoadley, Bishop of Bangor, citado por J.C. Gray, The Nature and Sources of the Law, cit., p. 102. 
ser revocadas (v.g., los órganos constitucionales supremos respecto de ciertas cláusulas constitucionales ${ }^{65}$ ), pueden atribuir a los textos normativos cualquier significado que deseen, y es también verdad que por lo general el derecho confiere a estas interpretaciones "auténticas" efectos jurídicos directos (cuanto menos inter partes) ${ }^{66}$.

Pero, ¿cuál es el alcance, respecto del significado de los textos normativos, de este enunciado descriptivo (verdadero)? Desde mi punto de vista, ninguno. Sobre la base de este enunciado la única cosa que puede ser sostenida con seguridad es que ciertas autoridades interpretativas pueden de facto ignorar el significado pre-existente de los textos normativos (si un significado tal existe). Pero esto no es un argumento a favor de la tesis central del escepticismo radical, a saber, que no existe ningún significado antes de la interpretación. Y, hasta donde sé, los filósofos del derecho no han todavía ofrecido otro argumento que permita defender con seriedad esa tesis ${ }^{67}$.

${ }^{65} \mathrm{M}$. Troper, "Les contraintes de l'argumentation juridique dans la production des normes", en O. Pfersmann, G. Timsit (eds.), Raisonnement juridique et interprétation, Publications de la Sorbonne, Paris, 2001, pp. 35 ss.; M. Troper, "L'interprétation constitutionnelle", en F. Mélin-Soucramanien (ed.), L'interprétation constitutionnelle, Dalloz, París, 2005.

${ }^{66}$ H. Kelsen, Pure Theory of Law, cit., p. 354.

${ }^{67}$ Es cierto que, fuera de la literatura filosófico jurídica, sería posible citar el así denominado escepticismo semántico kripkeano (S.A. Kripke, Wittgenstein on Rules and Private Language, Blackwell, Oxford, 1982). Sin embargo, si entiendo bien su argumento, Kripke simplemente muestra que «sentences that ascribe meanings are [...] non cognitive» (J.L. Coleman, B. Leiter, "Determinacy, Objectivity, and Authority", cit., p. 220). Desde mi punto de vista, por lo que se refiere a la interpretación decisoria en el ámbito jurídico, esto es cierto, pero no implica la tesis de la inexistencia de significado previo. Afirmar que una formulación normativa es ambigua (en sentido amplio) es diferente de afirmar que no posee ningún significado. Para algunas críticas al escepticismo semántico véase J.L. Coleman, B. Leiter, "Determinacy, Objectivity, and Authority", cit., p. 221 y ss.; J.J. Moreso, Legal Indeterminacy and Constitutional Interpretation, cit., p. 152 y ss. Para críticas a la "concepción particularista del significado" véase F. Schauer, Playing by the Rules, cit., p. 55 y ss. 
Adviértase que la tesis de la inexistencia de un significado previo equivale a sostener que ni siquiera el significado literal o prima facie -que se corresponde con el uso común- existe antes de la interpretación ${ }^{68}$. En otras palabras, la tesis implícitamente niega que existan reglas o convenciones o simplemente hábitos lingüísticos comúnmente compartidos. Además, si la tesis fuera cierta, volvería imposible cualquier análisis del lenguaje jurídico, ya que, al menos en ámbito jurídico, la empresa del análisis lingüístico (o lógico, o filosófico, etc.) se volvería un sinsentido (o carecería de interés). Resultaría imposible incluso distinguir entre enunciados con y sin sentido, entre enunciados ambiguos y no ambiguos, etc. Esto suena bastante implausible y, de hecho, contrario a la evidencia empírica, esto es, contrario a la evidencia de que existen reglas lingüísticas compartidas.

Aún más, la tesis de la inexistencia de un significado previo implica que todo enunciado que posea la forma lógica estándar de los enunciados interpretativos ("T significa S") cuenta como un genuino enunciado interpretativo. Por ejemplo, dada una cláusula constitucional que use la palabra "cámara", los enunciados interpretativos: (i) "'Cámara' significa sala o pieza principal de una casa", (ii) "'Cámara' significa cada uno de los cuerpos colegisladores", (iii) "'Cámara' significa una reunión de diseñadores de moda bien educados", serían interpretaciones rivales de una misma palabra e igualmente legítimas. Me parece claro que, por el contrario, (i) y (ii) constituyen atribuciones de significado -interpretaciones- que, si bien son distintas entre sí,

${ }^{68}$ El significado literal o claro es, podría decirse siguiendo a A. Marmor, una cuestión de comprensión y no de interpretación. Véase A. Marmor, Interpretation and Legal Theory, 2da ed., Hart Publishing, Oxford-Portland, 2005, p. 9 y ss.; A. Marmor, Positive Law and Objective Values, cit., p. 73 y ss. Sin embargo, es importante subrayar que cuando una persona atribuye a un texto normativo su significado literal (descartando otros significados disponibles) lleva a cabo una interpretación decisoria. El significado literal no posee ningún estatus privilegiado con relación a los demás significados posibles de un texto normativo. Véase P. Chiassoni, "On the Wrong Track: Andrei Marmor on Legal Positivism, Interpretation, and Easy Cases", en P. Comanducci, R. Guastini (eds.), Analisi e diritto 2007. Ricerche di giurisprudenza analitica, Giappichelli, Torino, 2008, esp. p. 149 y ss. 
todavía se encuentran dentro del rango de las posibilidades admitidas por el uso común del término (aun cuando la interpretación (i) sea bastante implausible), mientras que (iii) consiste en la creación de un significado totalmente nuevo. En otras palabras, no es que cualquier decisión sobre el significado -"Italia es una República democrática" significa "Italia es una monarquía absoluta"- constituya por eso solo una genuina interpretación, aun cuando imite la forma lógica ("T significa S") de estas últimas. Cada atribución de significado que caiga fuera del marco de los significados admisibles no constituye propiamente una "interpretación", sino más bien una creación de una norma nueva ${ }^{69}$.

El concepto de interpretación tácitamente asumido por la tesis de la inexistencia de significado previo es claramente demasiado amplio. Aplicada a la conversación de todos los días, volvería imposible distinguir entre la comprensión y el malentendido. Aplicada a las definiciones (cuya estructura lógica es idéntica a la de los enunciados interpretativos ${ }^{70}$ ), haría imposible distinguir entre definiciones de diccionario y estipulaciones. Y en particular, aplicada a la práctica jurídica, volvería imposible distinguir entre, por un lado, una decisión genuinamente interpretativa -la elección de uno de los significados disponibles dentro del "marco" de significados determinados mediante interpretación cognitiva- y, por otro lado, la creación por parte de los intérpretes de nuevos significados (de nuevas normas jurídicas).

La interpretación, por lo tanto, posee límites (conceptuales, no fácticos), en el sentido que no todo enunciado que pretende ser una interpretación puede razonablemente ser subsumido bajo el concepto de interpretación. Interpretar no consiste en atribuir cualquier significado, sino en atribuir uno de los significados que se encuentran dentro del rango de significados admisibles según (a) el uso común, (b) los métodos interpretativos en uso y, (c) las teorías dogmáticas. Tal como he sugerido

${ }^{69}$ R. Guastini, "Fundamentos de una teoría escéptica de la interpretación", en I. Lifante Vidal (ed.), Interpretación jurídica y teoría del Derecho, Palestra, Lima, 2010, p. 168.

${ }^{70}$ R. Guastini, "Interpretive Statements”, cit. 
más arriba, trazar límites al concepto de interpretación es necesario para poder distinguir entre la común atribución de significado a textos normativos -la interpretación decisoria propiamente dicha- y la genuina "legislación intersticial" por parte de juristas y jueces.

Es importante destacar, sin embargo, que asumir o admitir la existencia de un significado literal no equivale ni a decir que es el único significado (admisible), ni a decir que es el significado que los jueces (y los juristas) deben atribuir a los textos legales.

En primer lugar, que el significado literal no es el único significado posible de los textos normativos es un hecho. Los juristas y los jueces muy a menudo descartan el significado literal y, sin embargo, tales estrategias interpretativas no son consideradas sorprendentes $o$ irremediablemente inadmisibles por la comunidad interpretativa.

En segundo lugar, qué significado deba ser elegido -dentro del rango de los significados posibles- es una cuestión normativa, irrelevante desde el punto de vista de una teoría descriptiva de la interpretación.

En cualquier caso, el escepticismo ante las reglas no necesita comprometerse con un presupuesto tan robusto como la tesis de la inexistencia de significado previo a la interpretación. El verdadero fundamento del escepticismo ante las reglas es el reconocimiento de que es siempre posible interpretar un mismo texto de múltiples formas distintas (supra 1.2), asociado con la tesis de que no existe un criterio de verdad para los enunciados interpretativos (supra 2.3).

\subsection{El objeto de la disputa entre cognitivismo y escepticismo}

Según la teoría de la vigilia, parecería que la controversia entre cognitivismo y escepticismo ante las reglas recae sobre la interpretación dirigida a hechos, esto es, sobre el estatuto lógico de los enunciados subsuntivos $^{71}$ tales como "El hecho H es (o no es) un caso de homicidio",

71 "Subsumir" significa aplicar un predicado (en sentido lógico) a un individuo o a una clase, esto es, incluir un individuo dentro de una clase o incluir una clase dentro de otra clase más amplia. Por ejemplo: "El señor Giorgio Napolitano es un ciudadano italiano" equivale a incluir al Sr. Napolitano en 
El escepticismo ante las reglas replanteado

"Las patinetas son (o no son) vehículos", etc. ${ }^{72}$ Yo creo que esto no es así.

El objeto de la controversia son en realidad los enunciados interpretativos en sentido estricto, enunciados con la forma "T significa S" y que pertenecen a la interpretación dirigida a textos. Que los enunciados subsuntivos puedan tener valor de verdad es una tesis admisible (si bien bajo ciertas condiciones). Pero una buena teoría de la interpretación ha de proporcionar una respuesta convincente a la cuestión sobre la posibilidad de que los enunciados interpretativos tengan valor de verdad.

Aparentemente, la teoría de la vigilia ni siquiera distingue ${ }^{73}$ entre estos dos tipos de cuestiones. Por ejemplo, parece tratar como equivalentes cuestiones tales como: (a) si el enunciado normativo "No se admiten vehículos en el parque" se aplica a las patinetas o los triciclos, y (b) si el mismo enunciado se aplica a ambulancias o coches de bomberos ${ }^{74}$. Estas cuestiones, sin embargo, son de naturaleza bien diferente. La pregunta sobre las patinetas y los triciclos se refiere a la extensión del predicado "vehículo" y es, por lo tanto, una cuestión de interpretación "inconcreto" dirigida a hechos (de clasificación de hechos). La dificultad se presenta dado que no es pacífico que las patinetas o los triciclos sean vehículos en cuanto bien podrían, en cambio, ser juguetes. Mientras que la cuestión acerca de las ambulancias y los coches de bomberos es una

la clase de los ciudadanos italianos; "Todos los ciudadanos argentinos son americanos" equivale a incluir la clase de los ciudadanos argentinos en la clase más amplia de americanos.

72 Téngase en cuenta que la teoría de la vigilia es implicada por la teoría ampliamente aceptada según la cual únicamente los casos "difíciles" dan lugar a dudas interpretativas y a interpretación. Véase, por ejemplo, F. Schauer, Playing by the Rules, cit., p. 207 y s., p. 218 y s.

${ }^{73}$ Mientras que sí distingue, por ejemplo, W. Twining, D. Miers, How to Do Things with Rules, cit., p. 213 y ss.

74 «[R]ules [...] have an open texture, or have exceptions not exhaustively specifiable in advance»: H.L.A. Hart, The Concept of Law, cit., p. 135. Véase también T.A.O. Endicott, Vagueness, cit., p. 52 y ss., acerca de la que denomina "pragmatic vagueness" que, de hecho, equivale a la derrotabilidad. 
cuestión de interpretación "in abstracto" dirigida a textos ${ }^{75}$ y referida a la identificación misma de la norma. Ello en cuanto las ambulancias y los coches de bomberos son, desde todo punto de vista, vehículos. La pregunta por lo tanto es: ¿El texto expresa una norma referida a todos los vehículos de toda clase (sin excepciones) o expresa, en cambio, una norma derrotable sujeta a un número indefinido de excepciones implícitas (referidas, por ejemplo, a ambulancias y coches de bomberos)?

De hecho, la teoría de la vigilia -obsesionada por la vaguedad de los lenguajes naturales e interesada únicamente en la aplicación judicial ${ }^{76}$ no posee ninguna tesis definida sobre la ambigüedad (en el sentido amplio que he sugerido mediante ejemplos) de los textos jurídicos y la interpretación (principalmente dogmática) dirigida a textos. La razón de esto, supongo, es que la teoría de la vigilia da por descontado que la ambigüedad es un problema de interpretación meramente marginal y que no merece demasiada atención. No es un problema ya que, según una tácita asunción de la teoría, el contenido de significado de los textos normativos puede ser establecido con facilidad aplicando las reglas del lenguaje ordinario existentes. Así, si bien en algunos casos los jueces poseen discreción para decidir casos particulares, no poseen dicha discreción para identificar las reglas "in abstracto". La interpretación

${ }^{75}$ La conclusión según la cual las ambulancias no son admitidas presupone una interpretación literal que resulta de una norma que posee la forma lógica siguiente:

(x) $\mathrm{Vx} \rightarrow \mathrm{Php}$

(para todo $\mathrm{x}$, si $\mathrm{x}$ es un vehículo, su entrada en el parque está prohibida). La conclusión según la cual las ambulancias están admitidas supone una interpretación restrictiva cuyo resultado es una norma que contempla una excepción implícita:

$(\mathrm{x})(\mathrm{Vx} \wedge \neg \mathrm{Ax}) \rightarrow \mathrm{Php}$

(para todo $\mathrm{x}$, si $\mathrm{x}$ es un vehículo y no es una ambulancia, su entrada en el parque está prohibida). Esta notación pretende aclarar la diferente estructura lógica y los distintos contenidos normativos de las normas identificadas mediante las dos interpretaciones rivales.

${ }^{76}$ De hecho, la teoría de Hart se concentra en «the decisions whether a rule applies to a particular situation»: B. Bix, Law, Language, and Legal Determinacy, Clarendon, Oxford, 1993, p. 7. 
dirigida a textos es un acto de conocimiento y no de decisión ${ }^{77}$. Los enunciados interpretativos orientados a textos ("T significa S") son verdaderos o falsos.

Desafortunadamente, se trata de una posición bastante ingenua. Los jueces no siempre se atienen al significado literal $\mathrm{u}$ ordinario (a veces lo hacen pero muy a menudo no). Esta es la razón por la cual la teoría de la vigilia, si entendida como una teoría genuinamente descriptiva de la interpretación, es una teoría falsa o extremadamente incompleta -y, de hecho, una forma (tácita e inconfesada) de cognitivismo.

Sin embargo, la teoría de la vigilia puede ser entendida de un modo diverso. Puede -y quizás debe- ser entendida como una teoría normativa, que recomienda a los intérpretes seguir la reglas del lenguaje ordinario y descartar todo otro método interpretativo como también desatender cualquier teoría dogmática ${ }^{78}$. Pero, ¿por qué los intérpretes deberían hacer eso?

Al formular esta pregunta no estoy sugiriendo que no deban hacerlo. Simplemente asumo, una vez más, que las cuestiones normativas son irrelevantes para una teoría descriptiva de la interpretación y del significado en ámbito jurídico.

${ }^{77}$ Esta opinión está estrechamente vinculada con la asunción (ingenua) de que el lenguaje jurídico es parasitario del lenguaje natural y que, consecuentemente, la "comunicación jurídica" entre las autoridades normativas y los intérpretes es, a su vez, parasitaria de la comunicación ordinaria. Esto conduce a ignorar e incluso negar las particularidades de la práctica jurídica, tales como los conflictos de intereses, los métodos interpretativos de los juristas y la dogmática jurídica. Véase P. Chiassoni, "Interpretive Games. Statutory Construction through Gricean Eyes", cit.

${ }^{78}$ Además, me resulta realmente imposible advertir cómo sería posible ignorar las teorías dogmáticas cuando se interpreta términos jurídicos técnicos como "contrato", "responsabilidad", "negligencia", "propiedad", "posesión", "daño", "resarcimiento", etc. Aun más, la interpretación literal no permite llenar lagunas y resolver conflictos entre normas. 